\title{
Control of Normal Flow PDEs with ISS Properties
}

\author{
Angelo Alessandri, Patrizia Bagnerini, Christophe Prieur, Anna Rossi
}

\begin{abstract}
This paper establishes some input-to-state stability (ISS) properties w.r.t. in-domain process and measurement disturbances for systems described by a normal flow equation governed by an observer-based control scheme without knowledge of the spatial derivatives of the viscosity solution. The approach used to achieve the "a priori" ISS estimates of the solution is not based on Lyapunov arguments and assumes fixed boundary conditions. A one-dimensional case study is addressed by both analytical and numerical treatments, which show the effectiveness of the proposed approach.
\end{abstract}

\section{INTRODUCTION}

Input-to-State Stability (ISS) was developed by E. Sontag in the late eighties [1], [2], for systems described by ordinary differential equations, and has enabled the solution of numerous robust nonlinear control problems that were previously neither known nor addressed. No other foundational approach has reached comparable significance in the analysis of forced and interconnected nonlinear systems. Recently, a lot of efforts have been devoted to the extension of ISS to infinite-dimensional systems described by partial differential equations (PDEs). See recent textbook [3] for an introduction of this notion for infinite-dimensional systems, and [4] for a survey with some results on nonlinear ones, mainly semilinear parabolic or nonlinear delay systems. Despite the large literature, most of such results concern systems described by linear hyperbolic and parabolic PDEs, while very little is known about nonlinear PDEs as considered in this paper.

In this work, the robustness of a control scheme proposed in [5] for a class of nonlinear PDEs known as normal flow equations (NF, for short) is presented by showing its ISS properties in a suitable sense. NF equations are pretty popular in the research area of the so-called level set methods [6][8], which have been developed to describe the moving fronts for multi-dimensional applications such as fluid mechanics, image processing, and material science. Among other, the optimal control of heat equations is addressed in [9], [10], where the free boundaries are modelled by level sets. In [11], the use of approximation is pursued for the purpose of optimal control involving level sets. Feedback controllers for noise-free systems described by NF equations are presented in [5]. Modeling and identification of fire fronts by using level methods based on NF equations are considered in [12].

The stability of this class of nonlinear systems is in general nonrobust with respect to external vanishing disturbances,

A. Alessandri, P. Bagnerini. and A. Rossi are with the University of Genoa (DIME), Via all'Opera Pia 15, I-16145 Genoa, Italy (emails: angelo.alessandri@unige.it; patrizia.bagnerini@unige.it; anna.rossi@unige.it).

C. Prieur is with the University of Grenoble Alpes, CNRS, Grenoble-INP GIPSA-lab, F-38000, Grenoble, France (christophe.prieur@gipsa-lab.fr). His work has been partially supported by MIAI@Grenoble Alpes (ANR19-P3IA-0003) as illustrated by a specific example in this paper (see the numerical results section). Therefore a dedicated control theory needs to be developed for such nonlinear PDEs. In this context, the main objective of this work is to derive a suitable control design method ensuring an ISS property of the closed-loop system, in presence of external disturbance and output measurement noise. To do that, we rely on the wellposedness framework of [5] and extend the stability results presented therein by explicitly accounting for the presence of disturbances. It gives output feedback controllers so that the nonlinear closed-loop NF system is ISS for inputs defined by external disturbances and output measurement noise.

The importance of ISS in order to devise robust controllers for one-phase and two-phase Stefan models is highlighted in [13], where a number of real-world applications to processes with phase changes are presented. PDEs with moving boundaries have recently attracted a lot of attention for the capability to describe complex phenomena, which have been poorly investigated up to now (see also [14]). Clearly, the main difficulty is to find the appropriate ISS Lyapunov function under the most general assumptions [15].

The paper is organized as follows. Notation and preliminaries are described in Section II. The main result is presented in Section III. Section IV provides an example of asymptotically stable NF equation that is unstable in presence of a vanishing external disturbance. Moreover, applying the control design method of Section III yields an ISS closedloop system, as shown also by means of numerical simulations. Conclusion and prospect of future work are discussed in Section V.

\section{NotATION AND PRELIMINARIES}

Given a set $\Omega \subset \mathbb{R}^{q}, \bar{\Omega}, \partial \Omega, \mu(\Omega)$ denote its closure, boundary, and Lebesgue measure, respectively. The set $C^{0}(\Omega)$ is the class of continuous functions in $\Omega$, while $C^{1}(\Omega)$ is the class of continuous functions in $\Omega$ with continuous first-order derivatives. $B U C(\Omega)$ denotes the set of bounded uniformly continuous functions on $\Omega$. For any $T>0$, consider the Hamilton-Jacobi equation

$$
\phi_{t}(x, t)+H\left(x, t, \phi(x, t), \phi_{x}(x, t)\right)=0 \text { in } \Omega \times(0, T)
$$

where $\Omega$ is a bounded, smooth (e.g., with Lipschitz boundary), open set; $H: \Omega \times[0, T) \times \mathbb{R} \times \mathbb{R}^{q} \rightarrow \mathbb{R}$ is the Hamiltonian function; $\phi_{x}$ denotes the gradient of $\phi$ w.r.t. $x ; \phi_{0}(x) \in B U C(\bar{\Omega})$ denotes the initial condition in $\Omega ;$ boundary conditions are needed as well. The function $\phi_{1} \in$ $C^{0}(\Omega \times(0, T))$ is a viscosity subsolution of (1) if and only 
if there exists $v \in C^{1}(\Omega \times(0, T))$ such that

$$
v_{t}\left(x_{1}, t_{1}\right)+H\left(x_{1}, t_{1}, \phi_{1}\left(x_{1}, t_{1}\right), v_{x}\left(x_{1}, t_{1}\right)\right) \leq 0
$$

holds in $\left(x_{1}, t_{1}\right) \in \Omega \times(0, T)$ local maximum point of $\phi_{1}-v$. The function $\phi_{2} \in C^{0}(\Omega \times(0, T))$ is a viscosity supersolution of (1) if and only if there exists $v \in C^{1}(\Omega \times(0, T))$ such that

$$
v_{t}\left(x_{2}, t_{2}\right)+H\left(x_{2}, t_{2}, \phi_{2}\left(x_{2}, t_{2}\right), v_{x}\left(x_{2}, t_{2}\right)\right) \geq 0
$$

holds in $\left(x_{2}, t_{2}\right) \in \Omega \times(0, T)$ local minimum point of $\phi_{2}-v$.

If $\phi$ is a viscosity subsolution and a viscosity supersolution of (1), then $\phi$ is a viscosity solution of (1) (see [16, Definition II.1]). For any integer $n \geq 1$ and $x \in \mathbb{R}^{n}$, let $|x|:=\sqrt{\sum_{i=1}^{n} x_{i}^{2}} \cdot \mathcal{L}_{2}(\Omega)$ denotes the Hilbert space of square integrable functions $\gamma(\cdot, t): \Omega \rightarrow \mathbb{R}^{n}$ with norm $|\gamma(\cdot, t)|_{\mathcal{L}_{2}}:=\left(\int_{\Omega}|\gamma(x, t)|^{2} d x\right)^{1 / 2}<\infty$ for all $t \geq 0$. $\mathcal{H}^{1}(\Omega)$ is the Sobolev space of square integrable functions with square integrable first derivatives, i.e., $\mathcal{H}^{1}(\Omega):=\left\{\gamma \in \mathcal{L}_{2}(\Omega): \gamma_{x} \in \mathcal{L}_{2}(\Omega)\right\}$.

Let $\bar{\varphi} \in \mathcal{H}^{1}(\Omega)$ be an equilibrium of (1) if $H\left(x, t, \bar{\varphi}(x), \bar{\varphi}_{x}(x)\right)=0$ for any $t \geq 0$. Then, $\bar{\varphi}$ is said to be: (i) $\mathcal{L}_{2}$ stable if for all $\varepsilon>0$ there exists $\delta_{\varepsilon}>0$ such that $\left|\phi_{0}-\bar{\varphi}\right|_{\mathcal{L}_{2}}<\delta_{\varepsilon} \Rightarrow|\phi(\cdot, t)-\bar{\varphi}|_{\mathcal{L}_{2}}<\varepsilon$ for all $t \geq 0$; (ii) $\mathcal{L}_{2}$ asymptotically stable if it is stable and $\lim _{t \rightarrow+\infty}|\phi(\cdot, t)-\bar{\varphi}|_{\mathcal{L}_{2}}=0$; (iii) $\mathcal{L}_{2}$ exponentially stable if there exists $\lambda>0$ such that $|\phi(\cdot, t)-\bar{\varphi}|_{\mathcal{L}_{2}} \leq$ $c\left|\phi_{0}-\varphi\right|_{\mathcal{L}_{2}} \exp (-\lambda t)$ for some $c>0$ and all $t \geq 0$. If $|\phi(\cdot, t)-\bar{\varphi}|_{\mathcal{L}_{2}} \leq c \exp (-\lambda t)$ holds instead of the condition in (iii), i.e., without explicit dependence on $\left|\phi_{0}-\bar{\varphi}\right|_{\mathcal{L}_{2}}$ in the r.h.s., we simply say that $\phi$ converges exponentially to $\bar{\varphi}$ in the $\mathcal{L}_{2}$ sense. Finally, the Young inequality is the upper bound of the cross product of any couple of real numbers $a$ and $b$, as follows: $2 a b \leq a^{2}+b^{2}$.

\section{MAIN RESUlts}

In this section, we consider the control of a multidimensional NF equation affected by disturbances. More specifically, we deal with

$$
\left\{\begin{array}{l}
\phi_{t}(x, t)+f(x, t)\left|\phi_{x}(x, t)\right|=u(x, t)+w(x, t), \\
y(x, t)=\phi(x, t)+v(x, t)
\end{array}\right.
$$

in $\Omega \times(0, T)$, where $\Omega \subset \mathbb{R}^{q}, T>0, f$ is a fixed velocity field, $u$ is the in-domain input, $y$ is the output, $w$ denotes the in-domain disturbance, and $v$ accounts for output measurement noises. As pointed out in [5], the knowledge of the gradient of $\phi$ would facilitate the design of a stabilizing controller for (2) in the absence of noises, given by $u(x, t)=-k \phi(x, t)+f(x, t)\left|\phi_{x}(x, t)\right|$. Likewise in [5], we do not assume the knowledge of the spatial derivatives of the solution and rely on a Luenberger observer for the second term in the left-hand side of (2) (i.e., $f(x, t)\left|\phi_{x}(x, t)\right|$ ); this observer provides an estimate of $f(x, t)\left|\phi_{x}(x, t)\right|$ in such a way to apply the control given by

$$
u(x, t)=-k y(x, t)+f(x, t)\left|\hat{\phi}_{x}(x, t)\right|,
$$

where $k \in \mathbb{R}$ is the gain to be chosen and $\hat{\phi}(x, t)$ is the estimate of $\phi(x, t)$ given by the Luenberger observer

$\hat{\phi}_{t}(x, t)+f(x, t)\left|\hat{\phi}_{x}(x, t)\right|+k(\hat{\phi}(x, t)-y(x, t))=u(x, t)$.

The system based on the NF equation (2) entails the solution of the following boundary-value problem upon the application of a feedback law (this motivates the little abuse of notation concerning $u$ ):

$$
\left\{\begin{array}{l}
\phi_{t}(x, t)+f(x, t)\left|\phi_{x}(x, t)\right|=u(x, t, \phi(x, t))+w(x, t) \\
\quad \text { in } \Omega \times(0, T), \\
y(x, t)=\phi(x, t)+v(x, t) \text { in } \bar{\Omega} \times(0, T), \\
\phi(x, t)=\varphi(x) \text { in } \partial \Omega \times(0, T), \\
\phi(x, 0)=\phi_{0}(x) \text { in } \Omega,
\end{array}\right.
$$

where $\varphi \in B U C(\partial \Omega), \phi_{0} \in B U C(\bar{\Omega})$ and $f \in B U C(\bar{\Omega} \times$ $[0, T))$ is a known function acting as velocity field. Compared to (1), the Hamiltonian is given by $H(x, t, s, p)=$ $f(x, t)|p|-u(x, t, s)-w(x, t)$. The existence and uniqueness of viscosity solutions to (5) are guaranteed by the following assumptions: $w \in B U C(\bar{\Omega} \times[0, T)), u \in B U C(\bar{\Omega} \times[0, T) \times$ $\mathbb{R}), u$ nonincreasing w.r.t. $s$ for each $x \in \bar{\Omega}, t \in[0, T)$, $v \in \operatorname{BUC}(\bar{\Omega} \times[0, T))$, as well as $f, u, w$ and $v$ Lipschitz w.r.t. $x \in \Omega$. Thus, we can apply [17, Theorem 7.1, p. 82] since assumption (H1) is verified for $f, u, w, v$ Lipschitz w.r.t. $x \in \Omega$, and (H2) holds with $\gamma_{R}=0$, as $u$ is nonincreasing w.r.t. $s$ for each $x \in \bar{\Omega}, t \in[0, T)$. Concerning the observer (4), we have to deal with the problem

$$
\left\{\begin{array}{c}
\hat{\phi}_{t}(x, t)+f(x, t)\left|\hat{\phi}_{x}(x, t)\right|+k(\hat{\phi}(x, t)-\phi(x, t)) \\
\quad-k v(x, t)=u(x, t, \phi(x, t)) \text { in } \Omega \times(0, T), \\
\hat{\phi}(x, t)=\varphi(x) \text { in } \partial \Omega \times(0, T), \\
\hat{\phi}(x, 0)=\hat{\phi}_{0}(x) \text { in } \Omega,
\end{array}\right.
$$

for any $T>0$ and with $\hat{\phi}_{0} \in B U C(\bar{\Omega}), f$ and $u$ satisfying the same assumptions considered for (5), $\phi_{0}$ Lipschitz in $\bar{\Omega}$, and $\phi$ solution of (5). The problem (6) has a unique solution $\hat{\phi} \in \operatorname{BUC}(\bar{\Omega} \times[0, T))$ since (i) $\phi_{0}$ is Lipschitz in $\bar{\Omega}$, the unique solution $\phi$ of (5) is Lipschitz in $\bar{\Omega} \times[0, T)$ (see, e.g., [18]), and (ii) the Hamiltonian given by $H(x, t, s, p)=$ $f(x, t)|p|+k s-u(x, t, s)-k \phi(x, t)-k v(x, t)$ satisfies the conditions of existence stated in [17].

The following result connects the solution of (5) to the sub and supersolutions of (6).

Lemma 1: For any $T>0$, let $\phi$ be the viscosity solution of (5) with $\phi_{0}$ Lipschitz in $\bar{\Omega}$. Then, for any $\alpha \geq 1$,

$$
\begin{aligned}
\hat{\phi}_{1}(x, t) & :=\phi(x, t)+c e^{-\alpha t}-\sup _{\bar{\Omega} \times[0, T)}|w(x, t)| \\
& -k \sup _{\bar{\Omega} \times[0, T)}|v(x, t)| \in B U C(\bar{\Omega} \times[0, T))
\end{aligned}
$$

with $c<0$ and

$$
\hat{\phi}_{2}(x, t):=\phi(x, t)+d e^{-\alpha t}+\sup _{\bar{\Omega} \times[0, T)}|w(x, t)|
$$




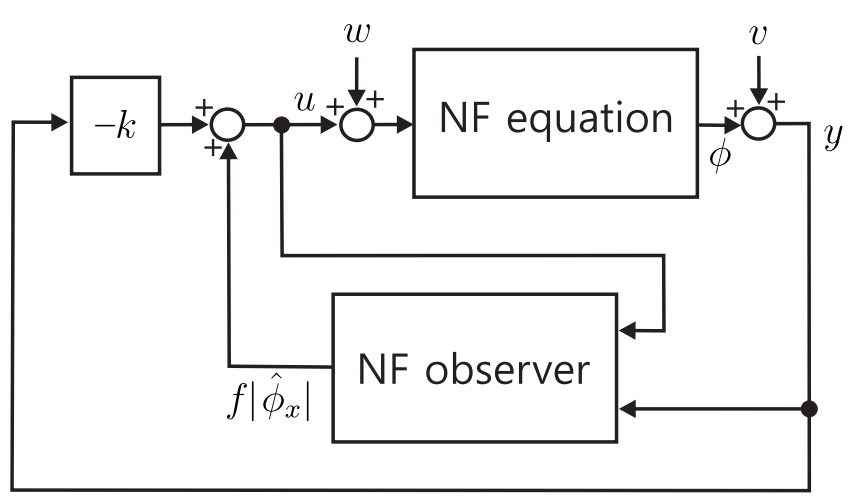

Fig. 1. Sketch of the control loop.

$$
+k \sup _{\bar{\Omega} \times[0, T)}|v(x, t)| \in B U C(\bar{\Omega} \times[0, T))
$$

with $d>0$ are a viscosity subsolution and a viscosity supersolution of (6) with $k \geq \alpha$, respectively.

The proof of the previous lemma is given in Appendix. For the proposed control (shown in Fig. 1) the following ISS property holds. This is the main result of this paper (proven in appendix section).

Theorem 1: For the closed-loop system given by (2), (3), and (4) under the assumptions stated in the formulations of the problems (5) and (6), if $k \geq 1$ the solutions $\phi, \hat{\phi} \in$ $\operatorname{BUC}(\bar{\Omega} \times[0, T))$ for all $T>0$ satisfy the following bound

$$
\begin{aligned}
& |\phi(\cdot, t)|_{\mathcal{L}_{2}} \leq \sqrt{2}\left(\mu(\Omega)^{1 / 2} \beta+|\hat{\phi}(\cdot, 0)|_{\mathcal{L}_{2}}\right) e^{-k t} \\
& +\sqrt{2} \mu(\Omega)^{1 / 2}\left(\sup _{\bar{\Omega} \times[0, T)}|w(x, t)|+k \sup _{\bar{\Omega} \times[0, T)}|v(x, t)|\right),
\end{aligned}
$$

for all $t \in[0, T)$, where $\beta>0$ is such that $\left|\phi_{0}(x)-\hat{\phi}_{0}(x)\right| \leq$ $\beta$ for all $x \in \bar{\Omega}$.

Remark. Some observations are in order.

- It is worth noting that the above result is more stringent as compared with the stability condition for the noisefree case presented in [5, Theorem 2, p. 4], for which it is required $k>0$ instead of $k \geq 1$.

- As for nonlinear finite-dimensional control systems, there exist globally asymptotically stable NF equations that are unstable in presence of vanishing disturbance.

Next section will concern the presentation of the results obtained with a simple case study.

\section{A Simple OnE-Dimensional Example}

In this section, we evaluate the effectiveness of the proposed approach by dealing with a simple one-dimensional example, which is analytically proved not to be ISS by showing an unstable behavior when subject to a converging disturbance in the absence of control. Next, we will illustrate how to stabilize this system by using the proposed control scheme and show simulations performed in Matlab with the level set methods toolbox developed by Mitchell [19].

Note that ISS property does not hold in general for globally asymptotically stable NF equations. Consider, for example, the one-dimensional NF equation

$$
\phi_{t}(x, t)+f(x, t)\left|\phi_{x}(x, t)\right|=0 \text { in }(0,1) \times[0,+\infty)
$$

where

$$
f(x, t):=\frac{\lambda(x)}{(t+2) \log (t+2)} \quad \lambda(x):=\min (x, 1-x)
$$

with boundary conditions $\phi(0, t)=\phi(1, t)=0$ for all $t \geq 0$. One can prove that (10) is globally stable in the $\mathcal{L}_{2}$ sense by using a simple quadratic Lyapunov functional. In addition, it is straightforward to verify that (10) with initial condition $\phi(x, 0)=\lambda(x)$ over $[0,1]$ admits the viscosity solution

$$
\phi(x, t)=\frac{\log (2) \lambda(x)}{\log (t+2)},
$$

having an $\mathcal{L}_{2}$ norm that converges asymptotically to zero. However, in the presence of an external in-domain disturbance as described by

$$
\phi_{t}(x, t)+f(x, t)\left|\phi_{x}(x, t)\right|=w(x, t)
$$

with initial condition $\phi(x, 0)=\lambda(x)$ and

$$
w(x, t):=\frac{2 \lambda(x)}{\log (2)(t+2)}
$$

tending to zero asymptotically, the viscosity solution

$$
\phi(x, t)=\frac{\log (t+2) \lambda(x)}{\log (2)}
$$

is asymptotically divergent.

Let us address the problem to design the proposed control to (10) and analyze it by means of simulations. The solution of the problems (5) and (6) together with (3) were obtained on the interval $[0,1]$ discretized via a regular grid composed of 100 discretization points. Both (5) and (6) were approximated with numerical schemes for nonlinear hyperbolic equations. In particular, a total variation diminishing Runge-Kutta scheme of second order was used for time discretization and an upwind second-order essentially non-oscillatory scheme [20] was utilized for space approximation. In all simulations we chose initial condition $\phi_{0}(x)=\hat{\phi}_{0}(x)=\lambda(x)$ and boundary conditions $\varphi(0)=$ $\varphi(1)=0$ for all $t \geq 0$.

In the first collection of tests, $w$ was given by (13), whereas we set $v \equiv 0$. The graphs of $\phi(\cdot, t)$ for different values of $t \in[0,1.5]$ are reported in Fig. 2 for $k=0,0.4,1$, and 5. As expected, $\phi$ diverges for $k=0$. Note that, even if for $k=0.4$ the stability condition $k \geq 1$ is not fulfilled, a stable behavior is observed since $\phi$ increases for a certain time and then decreases. This result is not surprising since the stability condition we found is proved to be only sufficient and not necessary, thus presumably conservative to some extent. For $k=1$ and $k=5, \phi$ shows an asymptotically 

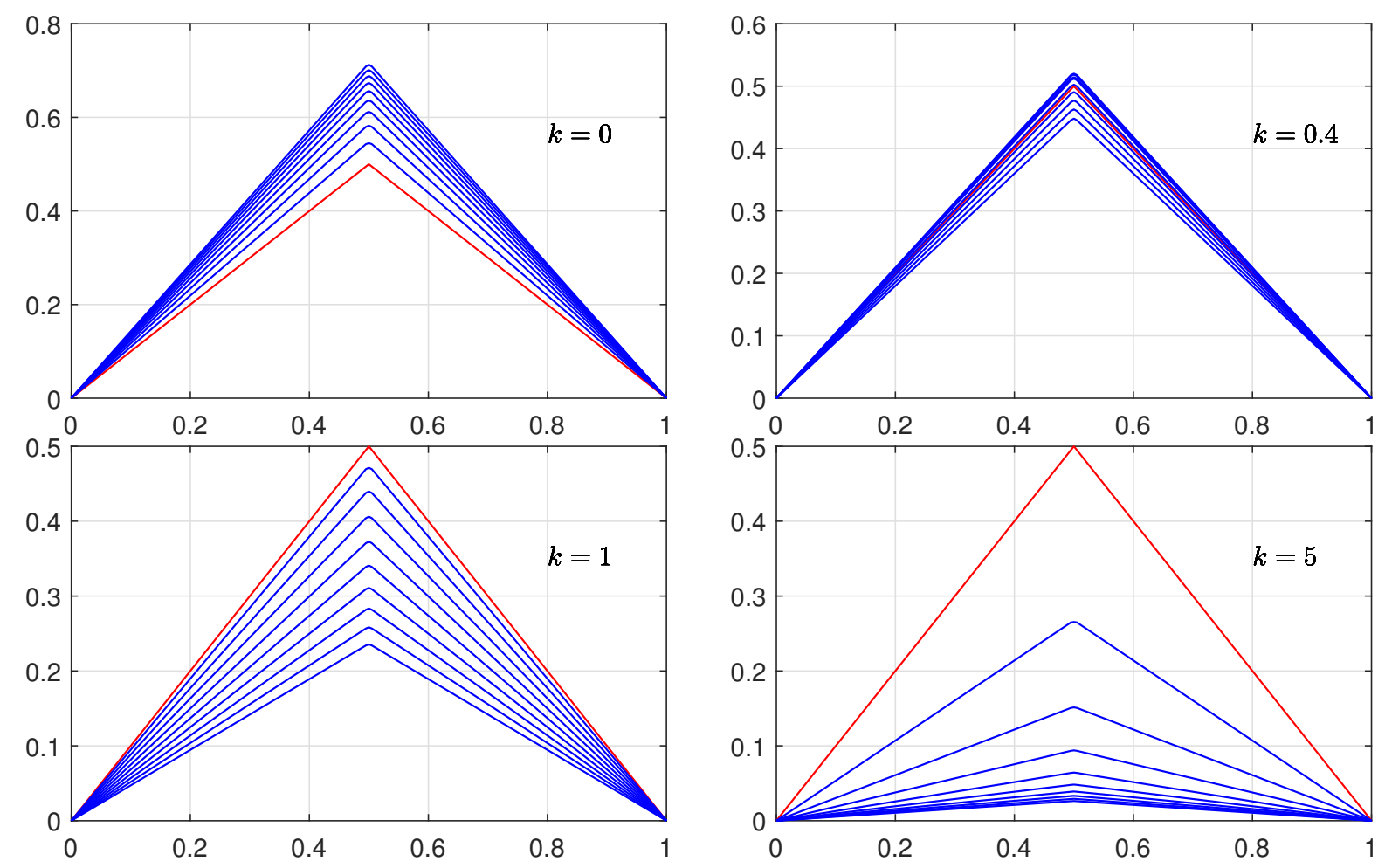

Fig. 2. Simulation results with in-domain disturbances (13) and control (3) for different values of $k=0,0.4,1,5$. The initial condition is depicted in red. For $k=0$ the solution diverges in accordance with what analytically shown, whereas a small overshoot occurs for $k=0.4$ since $\phi$ increases for a certain time and then decreases.

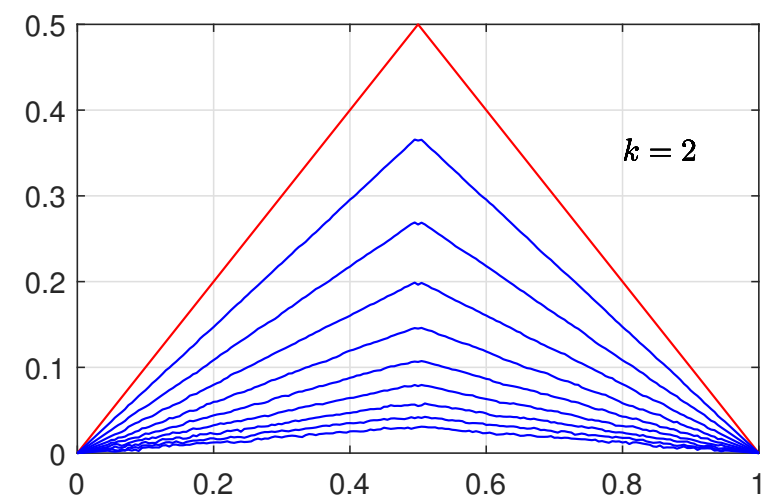

Fig. 3. Simulation result with control (3) $(k=2)$ and in-domain uniformly distributed random disturbances $w$ and $v$. The initial condition is depicted in red.

stable behavior to the origin as expected. In the second test illustrated in Fig. 3, random noises uniformly distributed in the ranges $[-0.08,0.08]$ were considered for both $w$ and $v$ with the control parameter $k$ set to 2 .

\section{CONCLUSION}

The paper extends some of the results presented in [5] by demonstrating that ISS bounds hold in a suitable sense for the considered observer-based control scheme for NF equations upon the choice of a sufficiently high gain. Such theoretical findings have been confirmed by simulation results. The relevance of this work concerns the potential application to the control of shape dynamics or moving fronts/interfaces, usually modelled by means of normal flow equations in two or higher dimensions, for which the present results hold. Thus, future efforts will be devoted to the control of more complicated systems such as airlift bioreactors (see, e.g., [21], [22]) or in general multi-phase flows described by Navier-Stokes equations combined with NF equations to account for moving interfaces (e.g., bubble dynamics for bioreactors), while providing robustness guarantees in terms of ISS.

\section{APPENDIX}

In this appendix we first prove Lemma 1, and then Theorem 1.

\section{A. Proof of Lemma 1}

First, we show that $\hat{\phi}_{1}$ is a viscosity subsolution of (6). Let $\theta \in C^{1}(\Omega \times(0, T))$. Since

$\tilde{\theta}(x, t):=\theta(x, t)-c e^{-\alpha t}+\sup _{\bar{\Omega} \times[0, T)}|w(x, t)|+k \sup _{\bar{\Omega} \times[0, T)}|v(x, t)|$

is a $C^{1}$ function in $\Omega \times(0, T)$ and $\phi$ is the viscosity solution of (5), it follows that

$$
\begin{aligned}
& \tilde{\theta}_{t}\left(x_{1}, t_{1}\right)+f\left(x_{1}, t_{1}\right)\left|\tilde{\theta}_{x}\left(x_{1}, t_{1}\right)\right| \\
& \quad-u\left(x_{1}, t_{1}, \phi\left(x_{1}, t_{1}\right)\right)-w\left(x_{1}, t_{1}\right) \leq 0
\end{aligned}
$$

if $\left(x_{1}, t_{1}\right) \in \Omega \times(0, T)$ is a local maximum point of $\phi-\tilde{\theta}$. By definition of $\tilde{\theta}$, we obtain

$$
\theta_{t}\left(x_{1}, t_{1}\right)+c \alpha e^{-\alpha t_{1}}+f\left(x_{1}, t_{1}\right)\left|\theta_{x}\left(x_{1}, t_{1}\right)\right|
$$




$$
-u\left(x_{1}, t_{1}, \phi\left(x_{1}, t_{1}\right)\right)-w\left(x_{1}, t_{1}\right) \leq 0
$$

and hence, using (7),

$$
\begin{aligned}
& \theta_{t}\left(x_{1}, t_{1}\right)+f\left(x_{1}, t_{1}\right)\left|\theta_{x}\left(x_{1}, t_{1}\right)\right|+\alpha\left(\hat{\phi}_{1}\left(x_{1}, t_{1}\right)-\phi\left(x_{1}, t_{1}\right)\right) \\
& -u\left(x_{1}, t_{1}, \phi\left(x_{1}, t_{1}\right)\right)+\alpha \sup _{\bar{\Omega} \times[0, T)}|w(x, t)|-w\left(x_{1}, t_{1}\right) \\
& +k \alpha \sup _{\bar{\Omega} \times[0, T)}|v(x, t)| \leq 0 .
\end{aligned}
$$

If $k \geq \alpha$, as $\hat{\phi}_{1}\left(x_{1}, t_{1}\right)-\phi\left(x_{1}, t_{1}\right)<0$, we obtain

$$
\begin{aligned}
& \theta_{t}\left(x_{1}, t_{1}\right)+f\left(x_{1}, t_{1}\right)\left|\theta_{x}\left(x_{1}, t_{1}\right)\right|+k\left(\hat{\phi}_{1}\left(x_{1}, t_{1}\right)\right. \\
& \left.-\phi\left(x_{1}, t_{1}\right)\right)-k v\left(x_{1}, t_{1}\right)-u\left(x_{1}, t_{1}, \phi\left(x_{1}, t_{1}\right)\right. \\
& \leq \theta_{t}\left(x_{1}, t_{1}\right)+f\left(x_{1}, t_{1}\right)\left|\theta_{x}\left(x_{1}, t_{1}\right)\right|+\alpha\left(\hat{\phi}_{1}\left(x_{1}, t_{1}\right)\right. \\
& \left.-\phi\left(x_{1}, t_{1}\right)\right)-u\left(x_{1}, t_{1}, \phi\left(x_{1}, t_{1}\right)\right)-k v\left(x_{1}, t_{1}\right) \\
& +\alpha \sup _{\bar{\Omega} \times[0, T)}|w(x, t)|-w\left(x_{1}, t_{1}\right)-\alpha \sup _{\bar{\Omega} \times[0, T)}|w(x, t)| \\
& +w\left(x_{1}, t_{1}\right)+k \alpha \sup _{\bar{\Omega} \times[0, T)}|v(x, t)|-k \alpha \sup _{\bar{\Omega} \times[0, T)}|v(x, t)| \\
& \leq-\alpha \sup _{\bar{\Omega} \times[0, T)}|w(x, t)|+w\left(x_{1}, t_{1}\right) \\
& -k\left(v\left(x_{1}, t_{1}\right)+\alpha \sup _{\bar{\Omega} \times[0, T)}|v(x, t)|\right) \leq 0
\end{aligned}
$$

where the last inequality holds since $\alpha \geq 1$. Therefore, $\hat{\phi}_{1}$ is a viscosity subsolution of (6) since $\left(x_{1}, t_{1}\right)$ is a local maximum also for

$$
\begin{aligned}
\hat{\phi}_{1}-\theta & =\phi+c e^{-\alpha t}-\sup _{\bar{\Omega} \times[0, T)}|w(x, t)|-k \sup _{\bar{\Omega} \times[0, T)}|v(x, t)| \\
& -\theta=\phi-\tilde{\theta} .
\end{aligned}
$$

To prove that $\hat{\phi}_{2}$ is a viscosity supersolution of (6), let $\left(x_{2}, t_{2}\right) \in \Omega \times(0, T)$ be a local minimum point of $\hat{\phi}_{2}-\theta$, for all $\theta \in C^{1}(\Omega \times(0, T))$. Since

$$
\tilde{\theta}(x, t):=\theta(x, t)-d e^{-\alpha t}-\sup _{\bar{\Omega} \times[0, T)}|w(x, t)|-k \sup _{\bar{\Omega} \times[0, T)}|v(x, t)|
$$

is a $C^{1}$ function in $\Omega \times(0, T)$, $\phi$ is a viscosity supersolution of $(5)$ and $\left(x_{2}, t_{2}\right)$ is also a local minimum point of $\phi-\tilde{\theta}=$ $\hat{\phi}_{2}-\theta$, we have

$$
\begin{aligned}
& \tilde{\theta}_{t}\left(x_{2}, t_{2}\right)+f\left(x_{2}, t_{2}\right)\left|\tilde{\theta}_{x}\left(x_{2}, t_{2}\right)\right|- \\
& u\left(x_{2}, t_{2}, \phi\left(x_{2}, t_{2}\right)\right)-w\left(x_{2}, t_{2}\right) \geq 0 .
\end{aligned}
$$

Thus, by definition of $\tilde{\theta}$, it follows that

$$
\begin{aligned}
& \theta_{t}\left(x_{2}, t_{2}\right)+d \alpha e^{-\alpha t_{2}}+f\left(x_{2}, t_{2}\right)\left|\theta_{x}\left(x_{2}, t_{2}\right)\right| \\
& -u\left(x_{2}, t_{2}, \phi\left(x_{2}, t_{2}\right)\right)-w\left(x_{2}, t_{2}\right) \geq 0
\end{aligned}
$$

and, by (8), reads

$$
\begin{aligned}
& \theta_{t}\left(x_{2}, t_{2}\right)+f\left(x_{2}, t_{2}\right)\left|\theta_{x}\left(x_{2}, t_{2}\right)\right|+\alpha\left(\hat{\phi}_{2}\left(x_{2}, t_{2}\right)\right. \\
& \left.-\phi\left(x_{2}, t_{2}\right)\right)-\alpha \sup _{\bar{\Omega} \times[0, T)}|w(x, t)|-w\left(x_{2}, t_{2}\right) \\
& -u\left(x_{2}, t_{2}, \phi\left(x_{2}, t_{2}\right)\right)-k \alpha \sup _{\bar{\Omega} \times[0, T)}|v(x, t)| \geq 0 .
\end{aligned}
$$

If $k \geq \alpha$, as $\hat{\phi}_{2}\left(x_{2}, t_{2}\right)-\phi\left(x_{2}, t_{2}\right)>0$, we obtain

$$
\theta_{t}\left(x_{2}, t_{2}\right)+f\left(x_{2}, t_{2}\right)\left|\theta_{x}\left(x_{2}, t_{2}\right)\right|+k\left(\hat{\phi}_{2}\left(x_{2}, t_{2}\right)\right.
$$

$$
\begin{aligned}
& \left.-\phi\left(x_{2}, t_{2}\right)\right)-k v\left(x_{2}, t_{2}\right)-u\left(x_{2}, t_{2}, \phi\left(x_{2}, t_{2}\right)\right) \\
& \geq \theta_{t}\left(x_{2}, t_{2}\right)+f\left(x_{2}, t_{2}\right)\left|\theta_{x}\left(x_{2}, t_{2}\right)\right|+\alpha\left(\hat{\phi}_{2}\left(x_{2}, t_{2}\right)\right. \\
& \left.-\phi\left(x_{2}, t_{2}\right)\right)-u\left(x_{2}, t_{2}, \phi\left(x_{2}, t_{2}\right)\right)-\alpha \sup _{\bar{\Omega} \times[0, T)}|w(x, t)| \\
& -w\left(x_{2}, t_{2}\right)+\alpha \sup _{\bar{\Omega} \times[0, T)}|w(x, t)|+w\left(x_{2}, t_{2}\right)-k v\left(x_{2}, t_{2}\right) \\
& +k \alpha \sup _{\bar{\Omega} \times[0, T)}|v(x, t)|-k \alpha \sup _{\bar{\Omega} \times[0, T)}|v(x, t)| \\
& \geq \alpha \sup _{\bar{\Omega} \times[0, T)}|w(x, t)|+w\left(x_{2}, t_{2}\right)+k\left(\alpha \sup _{\bar{\Omega} \times[0, T)}|v(x, t)|\right. \\
& \left.-v\left(x_{2}, t_{2}\right)\right) \geq 0,
\end{aligned}
$$

namely, $\hat{\phi}_{2}$ is a viscosity supersolution of (6). This concludes the proof of Lemma 1.

\section{B. Proof of Theorem 1}

Before proving the main result, let us first state and prove the following intermediate result based on Lemma 1.

Lemma 2: For any $\alpha \geq 1$, there exists $\beta>0$ such that

$$
\begin{aligned}
|\tilde{\phi}(x, t)| & \leq \beta e^{-\alpha t}+\sup _{\bar{\Omega} \times[0, T)}|w(x, t)| \\
& +k \sup _{\bar{\Omega} \times[0, T)}|v(x, t)|, t \in[0, T)
\end{aligned}
$$

for all $T>0$, where $\tilde{\phi}(x, t):=\phi(x, t)-\hat{\phi}(x, t)$ and $\phi, \hat{\phi}$ are viscosity solutions of (5) and (6), respectively, with $k \geq \alpha$ and $\phi_{0}, \hat{\phi}_{0}$ Lipschitz in $\bar{\Omega}$.

Proof. Since $\phi_{0}, \hat{\phi}_{0} \in C^{0}(\bar{\Omega})$, there exists $\beta>0$ such that $\left|\phi_{0}(x)-\hat{\phi}_{0}(x)\right| \leq \beta$. If $k \geq \alpha$, by Lemma 1 we can write that

$$
\begin{aligned}
\hat{\phi}_{1}(x, t) & =\phi(x, t)-\beta e^{-\alpha t}-\sup _{\bar{\Omega} \times[0, T)}|w(x, t)| \\
& -k \sup _{\bar{\Omega} \times[0, T)}|v(x, t)|
\end{aligned}
$$

and

$$
\begin{aligned}
\hat{\phi}_{2}(x, t) & =\phi(x, t)+\beta e^{-\alpha t}+\sup _{\bar{\Omega} \times[0, T)}|w(x, t)| \\
& +k \sup _{\Omega}|v(x, t)|
\end{aligned}
$$

are sub and supersolution of (4), respectively. Moreover,

$$
\begin{aligned}
& \hat{\phi}_{1}(x, 0)=\phi_{0}(x)-\beta-\sup _{\bar{\Omega} \times[0, T)}|w(x, t)| \\
& -k \sup _{\bar{\Omega} \times[0, T)}|v(x, t)| \leq \hat{\phi}_{0}(x) \leq \phi_{0}(x)+\beta \\
& +\sup _{\bar{\Omega} \times[0, T)}|w(x, t)|+k \sup _{\bar{\Omega} \times[0, T)}|v(x, t)|=\hat{\phi}_{2}(x, 0)
\end{aligned}
$$

for $x \in \Omega$ and

$$
\begin{aligned}
& \hat{\phi}_{1}(x, t)=\varphi(x)-\beta e^{-\alpha t}-\sup _{\bar{\Omega} \times[0, T)}|w(x, t)| \\
& -k \sup _{\bar{\Omega} \times[0, T)}|v(x, t)| \leq \hat{\phi}(x, t)=\varphi(x) \leq \hat{\phi}_{2}(x, t)=\varphi(x) \\
& +\beta e^{-\alpha t}+\sup _{\bar{\Omega} \times[0, T)}|w(x, t)|+k \sup _{\bar{\Omega} \times[0, T)}|v(x, t)|
\end{aligned}
$$


on $\partial \Omega \times(0, T)$. Hence, using [17, Theorem 5.1], it follows that, on $\Omega \times[0, T)$,

$$
\begin{aligned}
& \hat{\phi}_{1}(x, t)=\phi(x, t)-\beta e^{-\alpha t}-\sup _{\bar{\Omega} \times[0, T)}|w(x, t)| \\
& -k \sup _{\bar{\Omega} \times[0, T)}|v(x, t)| \leq \hat{\phi}(x, t) \leq \phi(x, t)+\beta e^{-\alpha t} \\
& +\sup _{\bar{\Omega} \times[0, T)}|w(x, t)|+k \sup _{\bar{\Omega} \times[0, T)}|v(x, t)|=\hat{\phi}_{2}(x, t)
\end{aligned}
$$

and

$$
\begin{aligned}
|\hat{\phi}(x, t)-\phi(x, t)| & \leq \beta e^{-\alpha t}+\sup _{\bar{\Omega} \times[0, T)}|w(x, t)| \\
& +k \sup _{\bar{\Omega} \times[0, T)}|v(x, t)|, t \in[0, T)
\end{aligned}
$$

for all $T>0$, thus obtaining (14) and concluding the proof of Lemma 2.

Using Lemma 2, we are now in position to prove the main result.

Proof of Theorem 1. Since $\phi, \hat{\phi} \in B U C(\bar{\Omega} \times[0, T))$, from (14) it follows that

$$
\begin{aligned}
|\tilde{\phi}(\cdot, t)|_{\mathcal{L}_{2}}^{2} & =\int_{\Omega}|\tilde{\phi}(x, t)|^{2} d x \leq\left(\beta e^{-\alpha t}\right. \\
& \left.+\sup _{\bar{\Omega} \times[0, T)}|w(x, t)|+k \sup _{\bar{\Omega} \times[0, T)}|v(x, t)|\right)^{2} \mu(\Omega)
\end{aligned}
$$

and hence

$$
\begin{aligned}
|\tilde{\phi}(\cdot, t)|_{\mathcal{L}_{2}} & \leq \mu(\Omega)^{1 / 2}\left(\beta e^{-\alpha t}+\sup _{\bar{\Omega} \times[0, T)}|w(x, t)|\right. \\
& \left.+k \sup _{\bar{\Omega} \times[0, T)}|v(x, t)|\right)
\end{aligned}
$$

Using the feedback control (3), i.e.,

$$
u(x, t)=-k \phi(x, t)-k v(x, t)+f(x, t)\left|\hat{\phi}_{x}(x, t)\right|,
$$

in (6) we get $\hat{\phi}_{t}(x, t)=-k \hat{\phi}(x, t)$ and hence it is straightforward to prove the $\mathcal{L}_{2}$ exponential stability of $\hat{\phi}$ to zero (see [5, Theorem 2, p. 4]). Using such a property, the Young inequality

$$
\begin{aligned}
\phi(x, t)^{2} & =(\phi(x, t)-\hat{\phi}(x, t)+\hat{\phi}(x, t))^{2} \\
& \leq 2 \tilde{\phi}(x, t)^{2}+2 \hat{\phi}(x, t)^{2}
\end{aligned}
$$

and the inequality $\sqrt{a+b} \leq \sqrt{a}+\sqrt{b}$ for $a, b \geq 0$, it follows that

$$
\begin{aligned}
& |\phi(\cdot, t)|_{\mathcal{L}_{2}} \leq \sqrt{2}\left(\int_{\Omega} \tilde{\phi}(x, t)^{2} d x\right)^{1 / 2}+\sqrt{2}\left(\int_{\Omega} \hat{\phi}(x, t)^{2} d x\right)^{1 / 2} \\
& \leq \sqrt{2} \mu(\Omega)^{1 / 2}\left(\beta e^{-\alpha t}+\sup _{\bar{\Omega} \times[0, T)}|w(x, t)|\right. \\
& \left.+k \sup _{\bar{\Omega} \times[0, T)}|v(x, t)|\right)+\sqrt{2}\left(\int_{\Omega} \hat{\phi}(x, 0)^{2} e^{-2 k t} d x\right)^{1 / 2} \\
& =\sqrt{2}\left(\mu(\Omega)^{1 / 2} \beta e^{-\alpha t}+|\hat{\phi}(\cdot, 0)|_{\mathcal{L}_{2}} e^{-k t}\right)
\end{aligned}
$$

$+\sqrt{2} \mu(\Omega)^{1 / 2}\left(\sup _{\bar{\Omega} \times[0, T)}|w(x, t)|+k \sup _{\bar{\Omega} \times[0, T)}|v(x, t)|\right)$

for any $k \geq \alpha$ with $\alpha \geq 1$. Thus, the choice $\alpha=k \geq 1$ provides the tightest bound given by (9). This concludes the proof of Theorem 1.

\section{REFERENCES}

[1] E. Sontag, "Smooth stabilization implies coprime factorization," IEEE Trans. on Automatic Control, vol. 34, no. 4, pp. 435-443, 1989.

[2] E. Sontag, "Remarks on stabilization and input-to-state stability," in Proc. 28th Conference on Decision and Control, Tampa, Florida, 1989, pp. 1376-1378.

[3] I. Karafyllis and M. Krstic, Input-to-State Stability for PDEs, ser. Communications and Control Engineering. Springer, 2018.

[4] A. Mironchenko and C. Prieur, "Input-to-state stability of infinitedimensional systems: recent results and open questions," SIAM Review, vol. 62 , no. 3, p. 529-614, 2020.

[5] A. Alessandri, P. Bagnerini, M. Gaggero, and A. Rossi, "State and observer-based feedback control of normal flow equations," Automatica, vol. 117, p. 108980, 2020.

[6] S. Osher and J. Sethian, "Fronts propagating with curvature-dependent speed: algorithms based on Hamilton-Jacobi formulations," J. Comput. Phys., vol. 79, no. 1, pp. 12-49, 1988.

[7] J. Sethian, Level Set Methods and Fast Marching Methods, 2nd ed. Cambridge: Cambridge University Press, 1999, vol. 3.

[8] S. Osher and R. Fedkiw, Level set methods and dynamic implicit surfaces, ser. Applied Mathematical Sciences. New York: SpringerVerlag, 2003, vol. 153.

[9] M. Hinze and S. Ziegenbalg, "Optimal control of the free boundary in a two-phase Stefan problem," J. Comput. Phys., vol. 223, no. 2, pp. 657-684, 2007.

[10] M. Bernauer and R. Herzog, "Optimal control of the classical twophase Stefan problem in level set formulation," SIAM J. Sci. Comput., vol. 33, no. 1, pp. 342-363, 2011.

[11] A. Alessandri, P. Bagnerini, and M. Gaggero, "Optimal control of propagating fronts by using level set methods and neural approximations," IEEE Trans. Neural Networks and Learning Systems, vol. 30, no. 3, pp. 902-912, 2019.

[12] A. Alessandri, P. Bagnerini, M. Gaggero, and L. Mantelli, "Parameter estimation of fire propagation models using level set methods," Applied Mathematical Modelling, vol. 92, pp. 731-747, 2021.

[13] S. Koga and M. Krstic, Materials Phase Change PDE Control and Estimation: From Additive Manufacturing to Polar Ice, ser. Systems \& Control: Foundations \& Applications. Cham: Springer International Publishing AG, 2020.

[14] S. Koga and M. Krstic, "Towards implementation of PDE control for Stefan system: Input-to-state stability and sampled-data design," Automatica, vol. 127, p. 109538, 2021.

[15] C. Prieur and F. Mazenc, "ISS-Lyapunov functions for time-varying hyperbolic systems of balance laws," Mathematics of Control, Signals, and Systems, vol. 24, no. 1, pp. 111-134, 2012.

[16] G. Barles, "Existence results for first order Hamilton Jacobi equations," Annales de l'I.H.P Analyse non linèaire, vol. 1, no. 5, pp. 325-340, 1984.

[17] G. Barles, An Introduction to the Theory of Viscosity Solutions for First-Order Hamilton-Jacobi Equations and Applications. Berlin, Heidelberg: Springer Berlin Heidelberg, 2013, pp. 49-109.

[18] P. Lions, Generalized Solutions of Hamilton-Jacobi Equations. London: Pitman, 1982.

[19] I. Mitchell, "The flexible, extensible and efficient toolbox of level set methods," J. Scientific Comput., vol. 35, no. 2-3, pp. 300-329, 2008.

[20] R. Kimmel, Numerical Geometry of Images. New York: SpringerVerlag, 2004.

[21] P. Bagnerini, M. Neviani, and O. Paladino, "Simulation of the rising of gas bubbles in a pilot-scale external loop airlift photobioreactor," in SUPEHR19, Sustainable Polyenergy Generation and Harvesting Conference, ser. E3S Web of Conferences, vol. 113, 2019, pp. 1-7.

[22] M. Neviani, P. Bagnerini, and O. Paladino, "Gas bubble dynamics in airlift photo-bioreactors for microalgae cultivation by level set methods," Fuel, vol. 292, p. 120402, 2021. 